\title{
Application of Mutiple Linear Regression Analysis in Polymer Modified Mortar Quality Control
}

\author{
Sai-bing QIU ${ }^{1, a}$, Bo TANG ${ }^{2, b}$ \\ ${ }^{1,2}$ College of Mathematics and Computer Science,Hunan City University,Hunan Yiyang \\ 413000, China \\ accsuqsb@163.com, bapriltb@163.com
}

Keywords: polymer modified mortar, mutiple linear regression analysis,significance checking.

\begin{abstract}
This paper is to predict the effect that the mixing charging, mixing water, and admixture and temperature to the quality control of the polymer modified cement mortar using the regression analysis method.With the water cement ratio and ploymer-cement ratio of a kind of polymer modified cement mortar as the dependent variable, the compressive strengh and flexural strength on quality control as the independent variables, the regression equation is established and the prediction interval result is achieved,which tested that there are multiple linear relations between the quality control's aims and the factors affecting the PCM's quality.
\end{abstract}

\section{Introduction}

Polymer modified cement mortar(PCM) is a kind of new building material with bright perspective, which is made of cement, aggregate and to spread the water organic matter into the mix polymerization. PCM overcomes the disadvantages of the complicated ploymer dip mortar process, large consumption and high cost of ploymer in the polymer mortar. Polymer can be not only homopolymers with a single polymerization, but also two or more single polymerization polymerized copolymer. Polymer must cover in the particles of the cement with specific environment conditions, and to make cement body and aggregate form strong bond. Polymer network must be able to prevent tiny cracks happen, and to stop the expansion of the crack. This kind of mortar has excellent bonding, crack resistance, antifreeze, preventing seepage, anticorrosive, anti-chloride ion penetration, ageing resistance and corrosion resistance. It is suitable for steel structure and concrete structure surface make up strong and patching, for example the sea, sluices, waterfalls, port engineering, roads, bridges, metallurgy, chemical industry, industrial floor and civil construction etc.

In engineering practice, the PCM rmust meet a certain strength requirements, water-retaining, the bond strength, iquidity and low cost. There are six factors affecting of PCM .They are ploymer type, bone material, ratio of ploymer and powder temperature, fine particle and chemical component ${ }^{[1]}$. Domestic and foreign research show that there are a lot of uncertainties between above goal and six factors, and there was no clear rules too. At present we cannot still find a corresponding analytical equation which can reflect their dominant relationship. From the view point of mathematical statistics theory, when the data that reflect objective characteristics is small, the data has obvious randomness, along with the increase of the quantity of data, and the number of true data that close to the characteristics of objective things will creases, when the amount of data tend to be infinite, the maximum likelihood value of data whould tend to be true value .In this paper, the relation between the quality control's aims and the factors affecting the PCM's quality is processed using mathematical staistics. The aims is to find out one part of influence factors to specific objectives when other factors are relatively stable, establish their approximate multiple relationship expression, and using a computer to the quality control of $\mathrm{PCM}^{[2]}$.

\section{The methods to solve the problems}

Multiple linear regression mathematics model. 
If a dependent variable $y_{t}$ can be denoted the linear function of independent variables $\left(x_{t 1}, x_{t 2}, \cdots x_{t k}\right)$

approximately, at the same time, a linear function of parameters $b_{0}$ and $b_{j}$ (usually unknown), random error

for $\varepsilon_{t}$, then the mathematical model of the multivariate linear regression can be expressed as :

$y_{t}=b_{0}+b_{1} x_{t 1}+b_{2} x_{t 2}+\cdots+b_{k} x_{t k}+\varepsilon_{t},(t=1,2, \cdots n)$.

In the above model, $b_{0}$ is the constant, $b_{1}, b_{2}, \cdots b_{k}$ are partial regression coefficient, denote that when other independent variable holds the line, $x_{i j}$ increase or decrease one unit that mean variation of the dependent variable $y_{t}$. The residual $\varepsilon_{t}$ is independent and normally distributed with mean 0 and variance $\delta^{2}$, it equates to that for any independent variables $x_{t 1}, x_{t 2}, \cdots x_{t k}$, the dependent variable $y_{t}$ has the same variance, and obey to normally distribution ${ }^{[3]}$. When given a sample $\left(y_{t}, x_{t 1}, x_{t 2}, \cdots x_{t k}, t=1,2, \cdots n\right)$, the model can be expressed as the matrix

$$
\left[\begin{array}{c}
y_{1} \\
y_{2} \\
\vdots \\
y_{n}
\end{array}\right]=\left[\begin{array}{cccccc}
1 & x_{11} & \cdots & x_{1 j} & \cdots & x_{1 k} \\
1 & x_{21} & \cdots & x_{2 j} & \cdots & x_{2 k} \\
\vdots & \vdots & \cdots & \vdots & \cdots & \vdots \\
1 & x_{n 1} & \cdots & x_{n j} & \cdots & x_{n k}
\end{array}\right]\left[\begin{array}{c}
b_{0} \\
b_{1} \\
\vdots \\
b_{k}
\end{array}\right]+\left[\begin{array}{c}
\varepsilon_{1} \\
\varepsilon_{2} \\
\vdots \\
\varepsilon_{n}
\end{array}\right] \text {. }
$$

Denoted by $Y=X b+\varepsilon$. Our primary goal is to determine the best of parameters $b_{i}(i=0,1,2, \cdots k)$, such that the model predicts experimental values of the dependent variable as accurately as possible. As the general linear regression equation, in order to get the multiple linear regression parameter estimation value, the error sum square $\sum \varepsilon^{2}$ must be minimum. The least square method or maximum likelihood estimation method is used to solve the parameter(i.e. a minimum $\left.Q=\sum_{t=1}^{n}\left(y_{t}-b_{0}-b_{1} x_{t 1}-\cdots b_{k} x_{t k}\right)^{2}\right)$.Using maximum likelihood estimate, let

$$
\begin{aligned}
& \left\{\begin{array}{l}
\frac{\partial Q}{\partial b_{0}}=2 \sum_{t=1}^{n}\left(y_{t}-b_{0}-b_{1} x_{t 1}-\cdots b_{k} x_{t k}\right)=0 \\
\frac{\partial Q}{\partial b_{j}}=-2 \sum_{t=1}^{n}\left(y_{t}-b_{0}-b_{1} x_{t 1}-\cdots b_{k} x_{t k}\right) x_{t j}=0
\end{array}\right. \\
& j=1,2, \cdots k \text {.Solving equations,we can get estimated values of } b_{0}, b_{1}, \cdots b_{k} .
\end{aligned}
$$

\section{The experimental method and data.}

This paper adopts literature [4] available experimental material and the method,to make into $40 \mathrm{~mm} \times 40 \mathrm{~mm} \times 40 \mathrm{~mm}$ try body, maintened 24 hours under temperature for $20^{\circ} \mathrm{C}$ and RH90 \% air, then stripped, contiue to maintain 6 days in temperature for $20^{\circ} \mathrm{C}$ water. At last, under temperature for $20^{\circ} \mathrm{C}$ and RH65 \% air to be maintained 21 days, altogether 28 days ${ }^{[4-6]}$. According to GB17671/1999 determination of compressive strength and flexural strength of the polymer modified cement mortar, we get the following experiment data in table 1 .

Table 1. The mixing proportion and performance of the PCM

\begin{tabular}{lcccc}
\hline & $\begin{array}{c}\text { Polymer- cement } \\
\text { ratio } \%\end{array}$ & $\begin{array}{c}\text { Water-cement } \\
\text { ratio }\end{array}$ & $\begin{array}{c}\text { Compressive strength } \\
(\mathrm{Mpa})\end{array}$ & $\begin{array}{c}\text { Flexural strength } \\
(\mathrm{Mpa})\end{array}$ \\
\hline Pure cement mortar & 0 & 0.4 & 50.63 & 7.39 \\
$50 / 50-5$ & 5 & 0.4 & 45.31 & 8.59 \\
$50 / 50-10$ & 10 & 0.4 & 35.63 & 9.30 \\
$50 / 50-15$ & 15 & 0.4 & 22.21 & 7.30 \\
$50 / 50-20$ & 20 & 0.4 & 27.18 & 6.40 \\
Pure cement mortar & 0 & 0.5 & 42.40 & 7.11
\end{tabular}




\begin{tabular}{|c|c|c|c|c|}
\hline $50 / 50-5$ & 5 & 0.5 & 27.87 & 7.37 \\
\hline $50 / 50-10$ & 10 & 0.5 & 26.30 & 6.90 \\
\hline 50/50-15 & 15 & 0.5 & 29.60 & 6.70 \\
\hline $50 / 50-20$ & 20 & 0.5 & 24.27 & 5.10 \\
\hline
\end{tabular}

\section{Calculation method.}

This paper use the method of multiple regression analysis to deal with experimental data obtained in Matlab 6.5. When other conditions remain unchanged, to discuss the influences of different water-cement ratio and polymer cement ratio to the PCM's flexural strength and compressive strength. Matlab is an advanced scientific computing software, which has been widely used in many fields, such as algorithm development, data visualization, data analysis and numerical calculation interactive application etc. It has been generally recognized as one of the standard calculation software in the field of scientific computing.This paper design a simple program in Matlab6.5 environment to generate an M-file, which reads as:

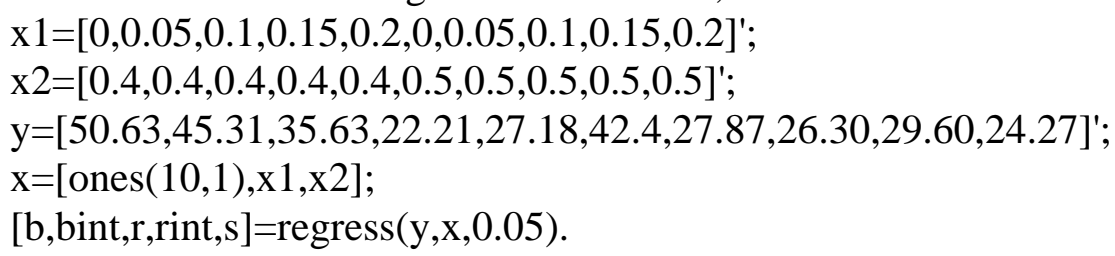

The estimated values of the parameteres are $\hat{b}_{1}=-104.53, \hat{b}_{2}=-61.04,95 \%$ confidence intervals are respectively (32.0136 110.1084), (-164.7815 -44.2785), (-146.2485 24.1685).The regression model is

$$
\hat{y}=71.061-104.53 x_{1}-61.04 x_{2}
$$

Regression parameters $R^{2}=0.7378, F=9.8494, P=0.0092 . R^{2}$ is the proportion of variation in the dependent variable that is predictable from the best linear combination of the independent variables. The closer $R^{2}$ is to one , the better that the model is . In the above model, $F>P, P<0.05$, wich shows that it is meaningful to the selection of the independent variables. Input rcoplot (r, rint) to analysis residual, the residuals and the leverage of the confidence interval in Figure 1 . We can seen from the lever diagram, all the residuals are uniformly distributed in the vicinity of 0:00. In other words, the data is not influential point, abnormal observation points. Taken together, the above regression results (regression function fit the curve or surface) is almost perfect.

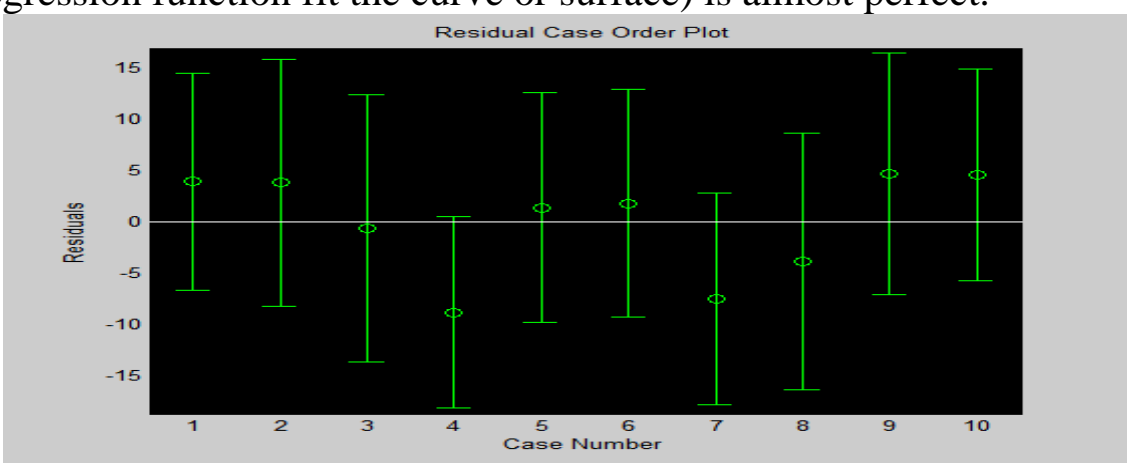

Figure 1

When research the influence about poly gray ratio and water-cement ratio against the flexural strength, the value dependent $y$ in table 1 for a replacement with value flexural strength, the regression model is

$$
\hat{y}=13.232-7.96 x_{1}-11.6 x_{2}
$$

Regression parameters $R^{2}=0.5530, F=4.3295, P=0.0597$, the results show $F>P$,but $p>0.05$,this doesn't meet the regression conditions. the residuals and the leverage of the confidence interval are shown in Figure 2.Easy to see that the third point is the outlier, remove outliers and re-regression calculations, regression model for the improved is as flow 


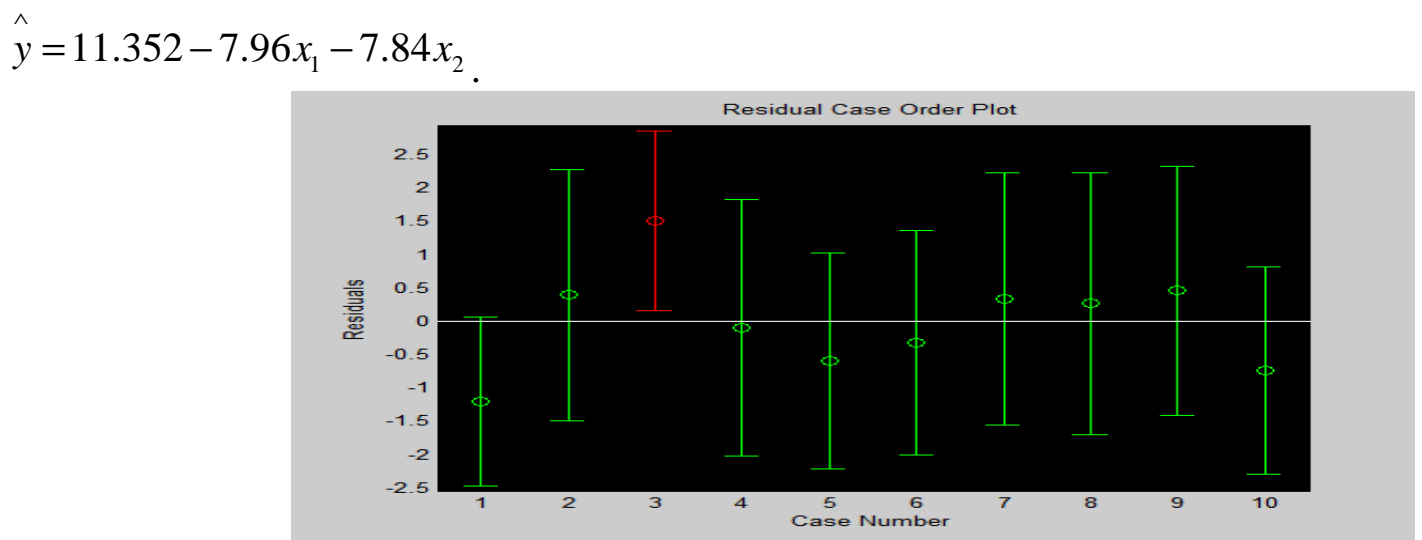

Figure 2

Regression parameters $R^{2}=0.6489, F=5.5449, P=0.0433$. It improved the regression model parameters $R^{2}$ and $F$ are increased, and $P<0.05$, which comply with the conditions of the regression model, the regression effect is more ideal.

\section{Conclusion}

If there are two or more independent variables in the regression model, called a multiple linear regression $^{[7]}$. In fact, a phenomenon often associated with a number of factors. The researches show that it is more effective and more realistic to predict or estimate the dependent variable by the optimal combination of multiple variables than with only one independent variable .

By the method of multiple linear regression model and numerical calculation with Matlab software, the computation results show that the flexural strength and compression strength of the modified cement mortar respectively have certain binary linear relationship with the water-cement ratio and polymer -cement ratio.From the binary linear regression model,we can get the corresponding one linear relationship too. At the case of water-cement ratio fixed, when the polymer cement ratio increase, the compressive strength of modified cement mortar decreased firstly ,then increased and trend to decreased finally,but the flexural strength increases first and then a downward trend. At the case of the polymer-cement ratio fixed, when the water-cement ratio was in the 0.4 , the compressive strength and flexural strength of the modified cement mortar were higher.

\section{References}

[1] J.X. Zhang,S.S.Jin,J,Zhang and D.Z.Wang,in:Study on Basic Characateristics of Ploymer Latex Modified Cement Mortar, volume 35 of Journal of Beijing UniversityofTechnology, Beijing University of Technology in Press (2009).

[2] T.F.Ollitraul, C.Gauthier, G.Clamen,in:Micro-structural Aspects in Ploymer- modified Cement, edited by S.Karen ,volume 28 of Cement and Concrete Research ,Elsevier Science in Press(1998).

[3] M.R.Wang,MATLAB and Scientific Computing(Publishing house of electronics industry, China 2003) [4] Y .Ohama,in:Ploymer-based Admixtures. volume 20 of Cem concr compos(1998)

[5] L.M. Liu, in: Application of regression analysis in the concrete energy prediction, edited by W.WANG. volume 23of Journal of Shengyang Institute of Aeronautical Engineering, Shengyang Institute of Aeronautical Engineering Press(2006)

[6] W.J.Zhao,H.X.Zhang,B.Y.Zhang,in:Efeects of Ploymer-cement Ratio and Water-Cement Ratio on Properties and Microstructure of Modified Mortars, volume 31 of Journal of Northeastern University, Northeastern University Press (2010).

[7] S.A.Wang,Mathematical Statistics(Beijing University of Science and Technology Publications, China 1995). 